\title{
On the Zeckendorf representation of smooth numbers
}

\author{
YANN BUGEAUd
}

\begin{abstract}
Among other results, we establish, in a quantitative form, that any sufficiently large integer cannot simultaneously be divisible only by very small primes and have very few digits in its Zeckendorf representation.
\end{abstract}

\section{Introduction and results}

The following general (and left intentionally vague) question was introduced and discussed in $[2,4]$ :

Do there exist arbitrarily large integers which have only small prime factors and, at the same time, few nonzero digits in their representation in some integer base?

The expected answer is no and modest steps in this direction have been made in [2, $4]$, by using a combination of estimates for linear forms in complex and $p$-adic logarithms of algebraic numbers. We refer to [2,4] for bibliographical references.

A similar question can be asked as well for the Zeckendorf representation [11] of integers having only small prime factors. Let $\left(F_{n}\right)_{n \geq 0}$ denote the Fibonacci sequence defined by $F_{0}=0, F_{1}=1$, and $F_{n+2}=F_{n+1}+F_{n}$ for $n \geq 0$. Every positive integer $N$ can be written uniquely as a sum

$$
N=\varepsilon_{\ell} F_{\ell}+\varepsilon_{\ell-1} F_{\ell-1}+\ldots+\varepsilon_{2} F_{2}+\varepsilon_{1} F_{1}
$$

with $\varepsilon_{\ell}=1, \varepsilon_{j}$ in $\{0,1\}$, and $\varepsilon_{j} \varepsilon_{j+1}=0$ for $j=1, \ldots, \ell-1$. This representation of $N$ is called its Zeckendorf representation. The number of digits of $N$ in its Zeckendorf representation is the number of positive integers $j$ for which $\varepsilon_{j}$ is equal to 1 . Recall that $F_{n}=\left(\gamma^{n}-\bar{\gamma}^{n}\right) / \sqrt{5}$ for $n \geq 0$, where $\gamma=(1+\sqrt{5}) / 2$ and $\bar{\gamma}=(1-\sqrt{5}) / 2$ is the Galois conjugate of $\gamma$. Since $\gamma$ is a unit, we cannot apply estimates for $p$-adic linear forms of logarithms as in $[2,4]$, thus the method developed in these papers cannot be straightforwardly adapted to this question.

2010 Mathematics Subject Classification: 11A63, 11J86, 11N25. 
The purpose of the present note is to give an alternative proof of the main results of [4], which rests only on estimates for linear forms in complex logarithms of algebraic numbers and can be extended to more general representations than $b$-ary representations, in particular to the Zeckendorf representation (and some other Ostrowski representations, see Section 4 for a short discussion). Moreover, it allows us also to extend some results of $[2,3]$. Note that an argument similar to ours can be found in [8].

Following [2], for an integer $k \geq 1$, we denote by $\left(F_{j}^{(k)}\right)_{j \geq 1}$ the sequence, arranged in increasing order, of all positive integers which have at most $k$ digits in their Zeckendorf representation. Said differently, $\left(F_{j}^{(k)}\right)_{j \geq 1}$ is the increasing sequence composed of the integers of the form

$$
F_{n_{h}}+\cdots+F_{n_{1}}, \quad n_{h} \geq n_{h-1}+2 \geq n_{h-2}+4 \geq \cdots \geq n_{1}+2 h-2, \quad n_{1} \geq 1, \quad 1 \leq h \leq k .
$$

In particular, the sequence $\left(F_{j}^{(1)}\right)_{j \geq 1}$ is the Fibonacci sequence $\left(F_{j}\right)_{j \geq 1}$.

Let $S=\left\{q_{1}, \ldots, q_{s}\right\}$ be a finite, non-empty set of distinct prime numbers. Let $n$ be a positive integer and write $n=A q_{1}^{r_{1}} \ldots q_{s}^{r_{s}}$, where $r_{1}, \ldots, r_{s}$ are non-negative integers and $A$ is an integer relatively prime to $q_{1} \ldots q_{s}$. We define the $S$-part $[n]_{S}$ of $n$ by

$$
[n]_{S}:=q_{1}^{r_{1}} \ldots q_{s}^{r_{s}} .
$$

Our first result shows that there are only finitely many integers which have a given number of digits in their Zeckendorf representation and whose prime divisors belong to a given finite set.

Theorem 1.1. Let $k$ be a positive integer and $\varepsilon$ a positive real number. Let $S$ be a finite, non-empty set of prime numbers. Then, we have

$$
\left[F_{j}^{(k)}\right]_{S}<\left(F_{j}^{(k)}\right)^{\varepsilon},
$$

for every sufficiently large integer $j$.

The case $k=1$ of Theorem 1.1 has already been established in [3]. Theorem 1.1 implies that, for any given positive integer $k$, the greatest prime factor of $F_{j}^{(k)}$ tends to infinity with $j$. However, its proof, based on the $p$-adic Schmidt Subspace Theorem, does not allow us to estimate the speed with which this greatest prime factor tends to infinity with $j$. Fortunately, we are able to derive such an estimate by means of the theory of linear forms in logarithms of algebraic numbers.

For a positive integer $n$, let $P[n]$ denote its greatest prime factor, with the convention that $P[1]=1$. A positive real number $B$ being given, a positive integer $n$ is called $B$ smooth if $P[n] \leq B$.

Theorem 1.2. Let $S$ be a finite, non-empty set of prime numbers. Let $k \geq 1$ be an integer. Then, there exist effectively computable positive numbers $c_{1}$ and $j_{1}$, depending only on $k$ and $S$, such that

$$
\left[F_{j}^{(k)}\right]_{S} \leq\left(F_{j}^{(k)}\right)^{1-c_{1}}, \quad \text { for } j \geq j_{1} .
$$


Furthermore, for every positive real number $\varepsilon$, there exists an effectively computable positive number $j_{2}$, depending only on $k$ and $\varepsilon$, such that

$$
P\left[F_{j}^{(k)}\right]>\left(\frac{1}{k}-\varepsilon\right) \log \log F_{j}^{(k)} \frac{\log \log \log F_{j}^{(k)}}{\log \log \log \log F_{j}^{(k)}}, \quad \text { for } j>j_{2} .
$$

In particular, there exists an effectively computable positive integer $n_{0}$, depending only on $k$ and $\varepsilon$, such that any integer $n>n_{0}$ which is

$$
\left(\frac{1}{k}-\varepsilon\right)(\log \log n) \frac{\log \log \log n}{\log \log \log \log n}-\text { smooth }
$$

has at least $k+1$ digits in its Zeckendorf representation.

A much stronger lower bound for $P\left[F_{j}^{(1)}\right]$ follows from Stewart's work [10], namely

$$
P\left[F_{j}^{(1)}\right]>j \exp (\log j / 104 \log \log j), \quad \text { for } j \text { large enough. }
$$

The analogues of Theorems 1.2 and 1.3 and of Corollaries 1.4 and 1.5 of [4] also hold for the Zeckendorf representation instead of the base- $b$ representation, as a consequence of Lemma 3.2 below. We state as Theorems 1.3 and 1.4 the statements analogous to Corollaries 1.4 and 1.5 of [4].

Theorem 1.3. Let $b \geq 2$ be an integer. There exists an effectively computable positive integer $n_{0}$ such that any integer $n>n_{0}$ satisfies the following three assertions. If $n$ is

$$
\frac{\log \log n}{2 \log \log \log \log n} \text {-smooth, then } n \text { has at least } \log \log \log n
$$

digits in its Zeckendorf representation. If $n$ is

$$
\sqrt{\log \log n \frac{\log \log \log n}{\log \log \log \log n}}-\text { smooth, then } n \text { has at least } \frac{1}{3} \sqrt{\log \log n \frac{\log \log \log n}{\log \log \log \log n}}
$$

digits in its Zeckendorf representation. If $n$ is

$$
\frac{1}{2} \log \log \log n \frac{\log \log \log \log n}{\log \log \log \log \log n} \text {-smooth, then } n \text { has at least } \frac{\log \log n}{2 \log \log \log n}
$$

digits in its Zeckendorf representation.

Let $S$ be a finite, non-empty set of prime numbers. A rational integer is an integral $S$-unit if all its prime factors belong to $S$. Proceeding as in [4], we can deduce from Lemma 3.2 below a lower bound for the number of digits in the Zeckendorf representation of integral $S$-units. 
Theorem 1.4. Let $S$ be a finite set of prime numbers. Then, for any positive real number $\varepsilon$, there exists an effectively computable positive integer $n_{0}$, depending only on $S$ and $\varepsilon$, such that any integral $S$-unit $n$ greater than $n_{0}$ has more than

$$
(1-\varepsilon) \frac{\log \log n}{\log \log \log n}
$$

digits in its Zeckendorf representation.

Our method allows us to extend Theorem 1.2 of [2] as follows. For a given integer $k \geq 2$, we denote by $\left(u_{j}^{(k)}\right)_{j \geq 1}$ the sequence, arranged in increasing order, of all positive integers which are not divisible by $b$ and have at most $k$ nonzero digits in their $b$-ary representation. Said differently, $\left(u_{j}^{(k)}\right)_{j \geq 1}$ is the ordered sequence composed of the integers $1,2, \ldots, b-1$ and those of the form

$d_{k} b^{n_{k}}+\ldots+d_{2} b^{n_{2}}+d_{1}, \quad n_{k}>\ldots>n_{2}>0, \quad d_{1}, \ldots, d_{k} \in\{0,1, \ldots, b-1\}, \quad d_{1} d_{k} \neq 0$.

Theorem 1.5. Let $b \geq 2$ and $k \geq 2$ be integers. Let $S$ be a finite, non-empty set of prime numbers. Then, there exist effectively computable positive numbers $c_{1}$ and $j_{1}$, depending only on $b, k$, and $S$, such that

$$
\left[u_{j}^{(k)}\right]_{S} \leq\left(u_{j}^{(k)}\right)^{1-c_{1}}, \quad \text { for every } j \geq j_{1} .
$$

Theorem 1.5 was proved in [2] for $k=2,3$ only.

We also get a (very slightly) weaker result than Theorem 1.1 of [4], namely, for any fixed $k \geq 3$, the lower bound

$$
P\left[u_{j}^{(k)}\right]>\left(\frac{1}{k-1}-\varepsilon\right) \log \log u_{j}^{(k)} \frac{\log \log \log u_{j}^{(k)}}{\log \log \log \log u_{j}^{(k)}}
$$

holds for any sufficiently large integer $j$. Note that $k-1$ is replaced by $k-2$ in Theorem 1.1 of $[4]$.

The paper is organized as follows. Theorem 1.1 is proved in Section 2 and Theorems 1.2 to 1.5 are established in Section 3. The final section contains some additional remarks.

\section{Proof of Theorem 1.1}

Let $K$ be an algebraic number field. Denote by $M_{K}$ the set of places of $K$. For $v$ in $M_{K}$, we choose a normalized absolute value $|\cdot|_{v}$ such that if $v$ is an infinite place, then

$$
|x|_{v}=|x|^{\left[K_{v}: \mathbf{R}\right] /[K: \mathbf{Q}]}, \text { for } x \in \mathbf{Q},
$$

while if $v$ is finite and lies above the prime $p$, then

$$
|x|_{v}=|x|_{p}^{\left[K_{v}: \mathbf{Q}_{p}\right] /[K: \mathbf{Q}]}, \text { for } x \in \mathbf{Q} .
$$


These absolute values satisfy the product formula

$$
\prod_{v \in M_{K}}|x|_{v}=1, \quad \text { for every non-zero } x \in K
$$

Moreover, if $x \in \mathbf{Q}$, then $\prod_{v \mid \infty}|x|_{v}=|x|$ and $\prod_{v \mid p}|x|_{v}=|x|_{p}$, where the products are taken over all infinite places of $K$, respectively all places of $K$ lying above the prime number $p$.

For later use, the height $h(x)$ of a non-zero $x$ in $K$ is defined by

$$
h(x)=\sum_{v \in M_{K}} \log \max \left\{1,|x|_{v}\right\}
$$

Let $T$ be a finite set of places of $K$, containing all the infinite places. Define the ring of $T$-integers of $K$ by

$$
O_{T}:=\left\{x \in K:|x|_{v} \leq 1 \text { for } v \in M_{K} \backslash T\right\}
$$

Further define

$$
H_{T}\left(x_{1}, \ldots, x_{n}\right):=\prod_{v \in T} \max \left\{\left|x_{1}\right|_{v}, \ldots,\left|x_{n}\right|_{v}\right\}, \text { for } x_{1}, \ldots, x_{n} \in O_{T}
$$

Our main tool is the following version of the $p$-adic Subspace Theorem, adapted from Theorem 3.1 .3 of [5].

Theorem 2.1. Let $K$ be an algebraic number field. Let $S$ be a finite set of prime numbers. Let $T$ be the finite set of places of $K$ composed of all the infinite places and all the places lying above the primes in $S$. For $v$ in $T$, let $L_{1 v}, \ldots, L_{n v}$ be linearly independent linear forms in $X_{1}, \ldots, X_{n}$ with coefficients in $K$. Let $\varepsilon>0$. Then the set of solutions of

$$
\prod_{v \in T}\left|L_{1 v}(\mathbf{x}) \cdots L_{n v}(\mathbf{x})\right|_{v} \leq H_{T}(\mathbf{x})^{-\varepsilon}, \quad \text { in } \mathbf{x} \in O_{K}^{n} \backslash\{\mathbf{0}\},
$$

is contained in a union of finitely many proper linear subspaces of $K^{n}$.

Now we proceed with the proof of Theorem 1.1 .

Let $k \geq 2$ be an integer and $\varepsilon$ a positive real number. Let $\mathcal{N}$ be the set of integer $k$-tuples $\left(n_{k}, \ldots, n_{1}\right)$ such that $n_{j}-n_{j-1} \geq 2$ for $j=2, \ldots, k, n_{1} \geq 1$, and

$$
\left[F_{n_{k}}+\cdots+F_{n_{1}}\right]_{S}>\left(F_{n_{k}}+\cdots+F_{n_{1}}\right)^{\varepsilon}
$$

Assume that $\mathcal{N}$ is infinite. Our aim is to apply Theorem 2.1 to get a contradiction. Let $\left(n_{k, i}, \ldots, n_{1, i}\right), i \geq 1$, denote an infinite subset of $\mathcal{N}$ ordered such that $n_{k, i}>n_{k, i-1}$ for $i \geq 2$. For technical reasons, which will be clear later, we would need to assume that

$$
\lim _{i \rightarrow+\infty}\left(n_{\ell, i}-n_{\ell-1, i}\right)=+\infty, \quad \ell=2, \ldots, k
$$


a condition which has no reason to be satisfied. Let us explain how one can proceed to get a similar assumption.

Observe that, for integers $m, \ell$ with $m>\ell>0$, we have

$$
F_{m}=F_{\ell} F_{m-\ell+1}+F_{\ell-1} F_{m-\ell}
$$

If there is an infinite subset $\mathcal{N}_{1}$ of $\mathbf{N}$ such that $n_{k, i}-n_{k-1, i}$ tends to infinity when $i$ tends to infinity along $\mathcal{N}_{1}$, then there is nothing more to do for the moment. Otherwise, there exist a positive integer $t$ and an infinite subset $\mathcal{N}_{2}$ of $\mathbf{N}$ such that $n_{k, i}-n_{k-1, i}=t$ for $i$ in $\mathcal{N}_{2}$. Then, instead of working with the $k$-tuple $\left(F_{n_{k, i}}, \ldots, F_{n_{1, i}}\right)$, we work with the $(k-1)$-tuple $\left(G_{n_{k-1, i}}, F_{n_{k-2, i}}, \ldots, F_{n_{1, i}}\right)$, where

$$
G_{n_{k-1, i}}=F_{t} F_{n_{k-1, i}+1}+F_{t-1} F_{n_{k-1, i}} .
$$

Proceeding like this, we can assume that there are an integer $h$, with $1 \leq h \leq k$, and an infinite subset $\mathcal{N}_{3}$ of $\mathbf{N}$ such that $n_{h, i}>\ldots>n_{1, i}, \quad i \in \mathcal{N}_{3}$,

$$
\begin{gathered}
F_{n_{k, i}}+\cdots+F_{n_{1, i}}=G_{n_{h, i}}+\cdots+G_{n_{1, i}}, \quad i \in \mathcal{N}_{3}, \\
{\left[G_{n_{h, i}}+\cdots+G_{n_{1, i}}\right]_{S}>\left(G_{n_{h, i}}+\cdots+G_{n_{1, i}}\right)^{\varepsilon}, \quad i \in \mathcal{N}_{3},}
\end{gathered}
$$

and

$$
\lim _{i \rightarrow+\infty}\left(n_{\ell, i}-n_{\ell-1, i}\right)=+\infty, \quad \ell=2, \ldots, h, \quad i \in \mathcal{N}_{3},
$$

where, for $j=1, \ldots, h$, we have

$$
G_{n_{j, i}}=a_{j} F_{n_{\ell(j), i}+1}+b_{j} F_{n_{\ell(j), i}}
$$

for non-negative integers $a_{j}, b_{j}$ and $\ell(j)$ in $\{1, \ldots, k\}$.

We are in position to apply Theorem 2.1.

We work in the quadratic field $K:=\mathbf{Q}(\sqrt{5})$. There are two complex embeddings, denoted by $|\cdot|_{\infty_{1}}$ and $|\cdot|_{\infty_{2}}$, numbered such that $|a+b \sqrt{5}|_{\infty_{1}}=|a+b \sqrt{5}|^{1 / 2}$ and $\mid a+$ $\left.b \sqrt{5}\right|_{\infty_{2}}=|a-b \sqrt{5}|^{1 / 2}$, for every rational numbers $a, b$. For $j=1, \ldots, h-1$, we consider the linear forms in $\mathbf{X}=\left(X_{1}, \ldots, X_{2 h}\right)$ defined by

$$
L_{2 j-1,1}(\mathbf{X}):=X_{2 j-1}-X_{2 j} / \sqrt{5}, \quad L_{2 j-1,2}(\mathbf{X}):=X_{2 j-1}
$$

and

$$
L_{2 j, 1}(\mathbf{X}):=X_{2 j}, \quad L_{2 j, 2}(\mathbf{X}):=X_{2 j}
$$

Set also

$$
L_{2 h-1,1}(\mathbf{X}):=X_{2 h-1}-X_{2 h} / \sqrt{5}, \quad L_{2 h-1,2}(\mathbf{X}):=X_{2 h-1},
$$

and

$$
L_{2 h, 1}(\mathbf{X}):=X_{1}+X_{3}+\ldots+X_{2 h-1}, \quad L_{2 h, 2}(\mathbf{X}):=X_{2}+X_{4}+\ldots+X_{2 h} .
$$


Let $T$ be the finite set of places of $K$ composed of all the infinite places and all the places lying above the primes in $S$. For every finite place $v$ in $T$, set

$$
L_{j, v}(\mathbf{X}):=X_{j}, \quad j=2, \ldots, 2 h, \quad L_{1, v}(\mathbf{X}):=X_{1}+X_{3}+\ldots+X_{2 h-1} .
$$

Recall that $\gamma=(1+\sqrt{5}) / 2$ and consider the points

$$
\mathbf{x}_{i}=\left(G_{n_{1, i}}, a_{1} \gamma^{n_{\ell(1), i}+1}+b_{1} \gamma^{n_{\ell(1), i}}, \ldots, G_{n_{h, i}}, a_{h} \gamma^{n_{\ell(h), i}+1}+b_{h} \gamma^{n_{\ell(h), i}}\right), \quad i \in \mathcal{N}_{3}
$$

By (2.2) and (2.3), we have

$$
\prod_{v \in T} \prod_{j=1}^{2 h}\left|L_{j, v}\left(\mathbf{x}_{i}\right)\right|_{v} \cdot \prod_{j=1}^{2 h}\left|L_{j, 1}\left(\mathbf{x}_{i}\right) L_{j, 2}\left(\mathbf{x}_{i}\right)\right|_{\infty_{1}}\left|L_{j, 1}\left(\mathbf{x}_{i}\right) L_{j, 2}\left(\mathbf{x}_{i}\right)\right|_{\infty_{2}} \leq H_{T}\left(\mathbf{x}_{i}\right)^{-\varepsilon / 2}
$$

for every $i$ large enough in $\mathcal{N}_{3}$.

It then follows from Theorem 2.1 that there exist $t_{1}, \ldots, t_{2 h}$ in $K$, not all zero, and an infinite set $\mathcal{N}_{4}$, contained in $\mathcal{N}_{3}$, such that (1.1)

$t_{2 h} G_{n_{h, i}}+t_{2 h-1}\left(a_{h} \gamma^{n_{\ell(h), i}+1}+b_{h} \gamma^{n_{\ell(h), i}}\right)+\cdots+t_{2} G_{n_{1, i}}+t_{1}\left(a_{1} \gamma^{n_{\ell(1), i}+1}+b_{1} \gamma^{n_{\ell(1), i}}\right)=0$,

for every $i$ in $\mathcal{N}_{4}$. Then, dividing $(2.5)$ by $G_{n_{h, i}}$ and letting $i$ tend to infinity along $\mathcal{N}_{4}$, we deduce from (2.4) that

$$
t_{2 h} G_{n_{h, i}}+t_{2 h-1}\left(a_{h} \gamma^{n_{\ell(h), i}+1}+b_{h} \gamma^{n_{\ell(h), i}}\right)=0
$$

for infinitely many $i$ in $\mathcal{N}_{4}$. Taking the Galois conjugate of (2.6), we obtain that $t_{2 h}=$ $t_{2 h-1}=0$. Continuing like this, we get $t_{1}=\ldots=t_{2 h}=0$, a contradiction. This shows that $\mathcal{N}$ cannot be infinite, thus (2.2) has only finitely many solutions. This completes the proof of Theorem 1.1.

\section{Proofs of Theorems 1.2 to $\mathbf{1 . 5}$}

The key tool for the proofs of Theorems 1.2 to 1.5 is the following immediate consequence of a theorem of Matveev [7]. The height $h$ of an algebraic number is defined in (2.1).

Theorem 3.1. Let $n \geq 2$ be an integer. Let $\alpha_{1}, \ldots, \alpha_{n}$ be non-zero algebraic real numbers. Let $D$ be the degree over $\mathbf{Q}$ of a number field containing $\alpha_{1}, \ldots, \alpha_{n}$. Let $A_{1}, \ldots, A_{n}$ be real numbers with

$$
\log A_{j} \geq \max \left\{h\left(\alpha_{j}\right), \frac{\left|\log \alpha_{j}\right|}{D}, \frac{0.16}{D}\right\}, \quad 1 \leq j \leq n .
$$

Let $b_{1}, \ldots, b_{n}$ be integers and set

$$
B^{\prime}=\max \left\{1, \max \left\{\left|b_{j}\right| \frac{\log A_{j}}{\log A_{n}}: 1 \leq j \leq n\right\}\right\} .
$$


Then, we have

$$
\log \left|\alpha_{1}^{b_{1}} \ldots \alpha_{n}^{b_{n}}-1\right|>-2 \times 30^{n+3} n^{4.5} D^{n+2} \log (\mathrm{e} D) \log A_{1} \ldots \log A_{n} \log \left(\mathrm{e} B^{\prime}\right)
$$

A key point in Theorem 3.1 is the presence of the factor $\log A_{n}$ in the denominator in the definition of $B^{\prime}$. It is crucial for getting a power saving in Theorems 1.2 and 1.5.

We first establish Theorem 1.5 and (1.1).

Let $b \geq 2$ be an integer. Below, the constants $c_{1}, c_{2}, \ldots$ are effectively computable and depend at most on $b$ and the constants $C_{1}, C_{2}, \ldots$ are absolute and effectively computable. Let $N$ be a positive integer greater than $b$ and $k$ the number of nonzero digits in its representation in base $b$. We assume that $b$ does not divide $N$, thus $k \geq 2$ and we write

$$
N=: d_{k} b^{n_{k}}+\cdots+d_{2} b^{n_{2}}+d_{1} b^{n_{1}}
$$

where

$$
n_{k}>\cdots>n_{2}>n_{1}=0, \quad d_{1}, \ldots, d_{k} \in\{1, \ldots, b-1\} .
$$

Let $q_{1}, \ldots, q_{s}$ denote distinct prime numbers written in increasing order. There exist nonnegative integers $r_{1}, \ldots, r_{s}$ and a positive integer $A$, coprime with $q_{1} \cdots q_{s}$, such that

$$
N=A q_{1}^{r_{1}} \cdots q_{s}^{r_{s}}
$$

In the case $A=1$, the following lemma is similar to Lemma 3.1 of [4].

Lemma 3.2. Under the above notation, we have

$$
n_{k} \leq\left(c_{1} C_{1}^{s} k\left(\prod_{i=1}^{s} \log q_{i}\right) \log \left(k \log q_{s}\right)\right)^{k-1} \max \{1, \log A\}
$$

Proof. Since

$$
\begin{aligned}
\Lambda_{k}:=\left|\left(A \prod_{i=1}^{s} q_{i}^{r_{i}}\right) d_{k}^{-1} b^{-n_{k}}-1\right|=\left|\left(\prod_{i=1}^{s} q_{i}^{r_{i}}\right) d_{k}^{-1} \frac{A}{b^{n_{k}}}-1\right| & =d_{k}^{-1} b^{-n_{k}} \sum_{h=1}^{k-1} d_{h} b^{n_{h}} \\
& \leq b^{1+n_{k-1}-n_{k}}
\end{aligned}
$$

we get

$$
\log \Lambda_{k} \leq-\left(n_{k}-n_{k-1}-1\right) \log b
$$

Set $A^{*}=\max \{A, \mathrm{e}\}$. Obviously, $\Lambda_{k}$ is non-zero. Since $r_{j} \log q_{j} \leq\left(n_{k}+1\right) \log b$ for $j=1, \ldots, s$, we deduce from Theorem 3.1 that

$$
\log \Lambda_{k} \geq-c_{2} C_{2}^{s}\left(\log q_{1}\right) \cdots\left(\log q_{s}\right)\left(\log A^{*}\right) \log \frac{n_{k}}{\log A^{*}}
$$


thus, by (3.1),

$$
n_{k}-n_{k-1}+\log A^{*} \leq c_{3} C_{3}^{s}\left(\log q_{1}\right) \cdots\left(\log q_{s}\right)\left(\log A^{*}\right) \log \frac{n_{k}}{\log A^{*}} .
$$

Likewise, for $j=2, \ldots, k-1$, we have

$$
\begin{aligned}
\Lambda_{j} & :=\left|\left(A \prod_{i=1}^{s} q_{i}^{r_{i}}\right) b^{-n_{j}}\left(d_{k} b^{n_{k}-n_{j}}+\ldots+d_{j}\right)^{-1}-1\right| \\
& =\left|\left(\prod_{i=1}^{s} q_{i}^{r_{i}}\right) b^{-n_{j}} \frac{A}{d_{k} b^{n_{k}-n_{j}}+\ldots+d_{j}}-1\right|=\frac{\sum_{h=1}^{j-1} d_{h} b^{n_{h}}}{\sum_{h=j}^{k} d_{h} b^{n_{h}}} \leq b^{1+n_{j-1}-n_{k}},
\end{aligned}
$$

thus,

$$
\log \Lambda_{j} \leq-\left(n_{k}-n_{j-1}-1\right) \log b .
$$

Since $\Lambda_{j}$ is non-zero, we deduce from Theorem 3.1 that

$$
\log \Lambda_{j} \geq-c_{4} C_{4}^{s}\left(\log q_{1}\right) \cdots\left(\log q_{s}\right)\left(n_{k}-n_{j}+\log A^{*}\right) \log \frac{n_{k}}{n_{k}-n_{j}+\log A^{*}} .
$$

Combining (3.3) and (3.4), we obtain

$$
n_{k}-n_{j-1} \leq c_{5} C_{5}^{s}\left(\prod_{i=1}^{s} \log q_{i}\right)\left(n_{k}-n_{j}+\log A^{*}\right) \log \frac{n_{k}}{n_{k}-n_{j}+\log A} .
$$

Consequently, we get

$$
n_{k}-n_{1} \leq n_{k}-n_{1}+\log A^{*} \leq c_{5} C_{5}^{s}\left(\prod_{i=1}^{s} \log q_{i}\right)\left(n_{k}-n_{2}+\log A^{*}\right) \log \frac{n_{k}}{\log A^{*}}
$$

and, for $j=3, \ldots, k-1$,

$$
n_{k}-n_{j-1}+\log A^{*} \leq c_{6} C_{6}^{s}\left(\prod_{i=1}^{s} \log q_{i}\right)\left(n_{k}-n_{j}+\log A^{*}\right) \log \frac{n_{k}}{\log A^{*}} .
$$

The combination of (3.2), (3.5), and (3.6) then gives

$$
n_{k}-n_{1} \leq\left(c_{7} C_{7}^{s}\left(\prod_{i=1}^{s} \log q_{i}\right)\right)^{k-1}\left(\log A^{*}\right)\left(\log \frac{n_{k}}{\log A^{*}}\right)^{k-1} .
$$

Since $n_{1}=1$, we get

$$
\left(\frac{n_{k}}{\log A^{*}}\right)^{1 /(k-1)} \leq c_{8} C_{8}^{s}\left(\prod_{i=1}^{s} \log q_{i}\right)(k-1)\left(\log \left(\frac{n_{k}}{\log A^{*}}\right)^{1 /(k-1)}\right),
$$


hence,

$$
n_{k} \leq\left(c_{9} C_{9}^{s} k\left(\prod_{i=1}^{s} \log q_{i}\right) \log \left(k \log q_{s}\right)\right)^{k-1}\left(\log A^{*}\right) .
$$

This establishes Lemma 3.2.

Since $N \leq\left(n_{k}+1\right) \log b$, Theorem 1.5 is a straightforward consequence of Lemma 3.2.

Now, we consider the Zeckendorf representation of $N$ and write

$$
F_{m_{k}}+\ldots+F_{m_{1}}=N=A q_{1}^{r_{1}} \cdots q_{s}^{r_{s}}
$$

with $m_{k} \geq m_{k-1}+2 \geq m_{k-2}+4 \geq \ldots \geq m_{1}+2 k-2$ and $m_{1} \geq 1$. Unlike for $b$-ary representations, we cannot assume that $m_{1}=1$.

Below, the constants $c_{10}, c_{11}, \ldots$ and $C_{10}, C_{11}, \ldots$ are absolute and effectively computable.

We establish the following analogue of Lemma 3.2.

Lemma 3.3. Under the above notation, we have

$$
m_{k} \leq\left(c_{10} C_{10}^{s} k\left(\prod_{i=1}^{s} \log q_{i}\right) \log \left(k \log q_{s}\right)\right)^{k} \max \{1, \log A\}
$$

Proof. We proceed as in the proof of Lemma 3.2, but we need to consider another linear form in logarithms to show that $m_{1}$ cannot be too large. Observe that

$$
0<\left|\sqrt{5} A q_{1}^{r_{1}} \cdots q_{s}^{r_{s}}-\gamma^{m_{1}}-\ldots-\gamma^{m_{k}}\right| \leq|\bar{\gamma}|^{m_{1}}+\ldots+|\bar{\gamma}|^{m_{k}} \leq \gamma
$$

so

$$
\begin{aligned}
\Lambda & :=\left|A q_{1}^{r_{1}} \cdots q_{s}^{r_{s}} \gamma^{-m_{1}}\left(1+\gamma^{m_{2}-m_{1}}+\ldots+\gamma^{m_{k}-m_{1}}\right)^{-1}-1\right| \\
& =\left|q_{1}^{r_{1}} \cdots q_{s}^{r_{s}} \gamma^{-m_{1}} \frac{A}{1+\gamma^{m_{2}-m_{1}}+\ldots+\gamma^{m_{k}-m_{1}}}-1\right|<\gamma^{-m_{k}+1} .
\end{aligned}
$$

Observe that there is an absolute positive constant $C$ such that

$$
h\left(1+\gamma^{m_{2}-m_{1}}+\ldots+\gamma^{m_{k}-m_{1}}\right) \leq C\left(m_{k}-m_{1}\right) .
$$

Applying Theorem 3.1, this gives

$$
m_{k} \leq c_{11} C_{11}^{s}\left(\prod_{i=1}^{s} \log q_{i}\right)\left(m_{k}-m_{1}+\log A^{*}\right) \log \frac{m_{k}}{\log A^{*}} .
$$

We consider the quantities analogous to the $\Lambda_{j}$ 's occurring in the proof of Lemma 3.2 and, before applying Theorem 3.1, we need to check that there are nonzero. To this end, we may proceed as follows. Let $j$ be an integer with $1 \leq j \leq k$. Assume that

$$
\sqrt{5} N=\gamma^{m_{k}}+\ldots+\gamma^{m_{j}}
$$


Take the Galois conjugate to get

$$
-\sqrt{5} N=\bar{\gamma}^{m_{k}}+\ldots+\bar{\gamma}^{m_{j}}
$$

Subtracting (3.8) from (3.7), we get

$$
2 N=F_{m_{k}}+\ldots+F_{m_{j}}
$$

a contradiction.

Since the exact analogues of (3.2) and (3.5) hold in our context, we proceed as in the proof of Lemma 3.2 to get

$$
\left(\frac{m_{k}}{\log A^{*}}\right)^{1 / k} \leq c_{12} C_{12}^{s}\left(\prod_{i=1}^{s} \log q_{i}\right) k\left(\log \left(\frac{m_{k}}{\log A^{*}}\right)^{1 / k}\right)
$$

and

$$
m_{k} \leq\left(c_{13} C_{13}^{s} k\left(\prod_{i=1}^{s} \log q_{i}\right) \log \left(k \log q_{s}\right)\right)^{k}\left(\log A^{*}\right) .
$$

This establishes Lemma 3.3.

The first statement of Theorem 1.2 is a straightforward consequence of Lemma 3.1. To obtain the other statements, as well as Theorems 1.3 and 1.4, we proceed exactly as in [4]. We omit the details.

\section{Further remarks}

Let $\alpha$ be an irrational real number whose continued fraction expansion is given by $\left[a_{0} ; a_{1}, a_{2}, \ldots\right]$. For $n \geq 0$, let $q_{n}$ be the denominator of the rational number $\left[a_{0} ; a_{1}, \ldots, a_{n}\right]$. Then (see Theorem 3.9.1 of [1] for a proof), every positive integer $N$ can be represented uniquely in the form

$$
N=d_{\ell} q_{\ell}+\ldots+d_{1} q_{1}+d_{0} q_{0},
$$

where the integers $d_{0}, \ldots, d_{\ell}$ satisfy the following three condiitons:

1. $0 \leq d_{0}<a_{0}$.

2. $0 \leq d_{i} \leq a_{i+1}$, for $i \geq 1$.

3. $\quad$ For $i \geq 1$, if $d_{i}=a_{i+1}$, then $d_{i-1}=0$.

This representation of $N$ is called its representation in the Ostrowski $\alpha$-numeration system. When $\alpha$ is the Golden Ratio, this is precisely the Zeckendorf representation of $N$.

For positive integers $b_{1}, \ldots, b_{s}$, Lenstra and Shallit [6] established that, if $\left(p_{n} / q_{n}\right)_{n \geq 1}$ denotes the sequence of convergents to

$$
\alpha=\left[0 ; \overline{b_{1}, \ldots, b_{s}}\right]
$$

then $q_{n+2 s}=t q_{n+s}-(-1)^{s} q_{n}$, for $n \geq 1$, where $t=p_{s-1}+q_{s}$. This shows that, for $h=0, \ldots, s-1$, the sequence $\left(q_{n s+h}\right)_{n \geq 0}$ satisfies a binary recurrence relation. More 
precisely, there exist $a_{h}$ in the quadratic field generated by $\left(t+\sqrt{t^{2}-4 \times(-1)^{s}}\right) / 2$ such that

$$
q_{n s+h}=a_{h}\left(\frac{t+\sqrt{t^{2}-4 \times(-1)^{s}}}{2}\right)^{n}+\bar{a}_{h}\left(\frac{t-\sqrt{t^{2}-4 \times(-1)^{s}}}{2}\right)^{n}, \quad n \geq 0
$$

where the bar denotes the Galois conjugate. A similar statement holds for every quadratic real number $\alpha$.

More generally, Theorems 1.1 to 1.4 are still valid when the Zeckendorf representation is replaced by the representation in the Ostrowski $\alpha$-numeration system, provided that $\alpha$ is a quadratic real number.

We have focused our attention to digital representations of integers. Actually, we can more generally consider finite sums of values of a given non-degenerate recurrence sequence of integers having a dominant root. Let

$$
u_{n}=f_{1}(n) \alpha_{1}^{n}+\ldots+f_{t}(n) \alpha_{t}^{n}, \quad n \geq 0
$$

be a nondegenerate recurrence sequence of integers having a dominant root. Here, $f_{1}, \ldots, f_{t}$ are polynomials with coefficients in the algebraic number field $K:=\mathbf{Q}\left(\alpha_{1}, \ldots, \alpha_{t}\right)$, for all integers $i, j$ with $1 \leq i<j \leq t$, the quotient $\alpha_{i} / \alpha_{j}$ is not a root of unity, and $\left|\alpha_{1}\right|>\left|\alpha_{j}\right|$, for $j=2, \ldots, t$. For example, the Fibonacci sequence has these properties.

For an integer $k \geq 1$, we denote by $\left(U_{j}^{(k)}\right)_{j \geq 1}$ the sequence, arranged in increasing order, of all the integers of the form

$$
u_{n_{k}}+\cdots+u_{n_{1}}, \quad n_{k}>\ldots>n_{1} .
$$

Let $S$ be a finite, non-empty set of prime numbers. We can proceed as in the proof of Theorem 1.2 in order to bound $\left[U_{j}^{(k)}\right]_{S}$ from above. A difficulty arises since we have to ensure that the quantities analogous to the $\Lambda_{j}$ 's are nonzero. This may require additional assumption on the sequence $\left(u_{n}\right)_{n \geq 0}$. We leave the details to an interested reader.

\section{References}

[1] J.-P. Allouche and J. Shallit, Automatic Sequences: Theory, Applications, Generalizations, Cambridge University Press, 2003.

[2] Y. Bugeaud, On the digital representation of integers with bounded prime factors, Osaka J. Math. 55 (2018), 315-324.

[3] Y. Bugeaud and J.-H. Evertse, S-parts of terms of integer linear recurrence sequences, Mathematika 63 (2017), 840-851.

[4] Y. Bugeaud and H. Kaneko, On the digital representation of smooth numbers, Math. Proc. Cambridge Philos. Soc. 165 (2018), 533-540. 
[5] J.-H. Evertse and K. Győry, Unit Equations in Diophantine Number Theory. Cambridge University Press, 2015.

[6] H. W. Lenstra and J. O. Shallit, Continued fractions and linear recurrences, Math. Comp. 61 (1993), 351-354.

[7] E. M. Matveev, An explicit lower bound for a homogeneous rational linear form in logarithms of algebraic numbers. II, Izv. Ross. Acad. Nauk Ser. Mat. 64 (2000), 125-180 (in Russian); English translation in Izv. Math. 64 (2000), 1217-1269.

[8] E. Mazumdar and S. S. Rout, Prime powers in sums of terms of binary recurrence sequences, Monatsh. Math. 189 (2019), 695-714.

[9] C. L. Stewart, On the representation of an integer in two different bases, J. reine angew. Math. 319 (1980), 63-72.

[10] C. L. Stewart, On divisors of Lucas and Lehmer numbers, Acta Math. 211 (2013), 291-314.

[11] E. Zeckendorf, Représentation des nombres naturels par une somme de nombres de Fibonacci ou de nombres de Lucas, Bull. Soc. Roy. Sci. Liège 41 (1972), 179-182.

Yann Bugeaud

Institut de Recherche Mathématique Avancée, U.M.R. 7501

Université de Strasbourg et C.N.R.S.

7 , rue René Descartes

67084 Strasbourg, FRANCE

e-mail : bugeaud@math.unistra.fr 